\title{
Pharmacist's Intervention Considering the Prognosis for a Terminal Cancer Patient: A Case Report
}

\author{
Masahiro Okada ${ }^{1}$, Kazuko Okazaki ${ }^{1}$, Keisuke Kimura ${ }^{2}$, Hiroki Sugihara ${ }^{1}$, \\ Fumiyoshi Murakami ${ }^{1}$, Shinya Okamoto ${ }^{1}$, Yoshinori Hoshino ${ }^{1}$, Yuka Goto ${ }^{1}$, Kengo Banshoya ${ }^{3}$, \\ Tadashi Onoda ${ }^{2}$, Eisuke Takei ${ }^{1}$, Shuso Takeda ${ }^{3}$ and Narumi Sugihara ${ }^{3, *}$ \\ 1 Department of Pharmacy Onomichi Municipal Hospital, Shintakayama, Onomichi, Hiroshima 3-1170-177, \\ Japan; okada.masahiro@omhp.jp (M.O.); okazaki.kazuko@omhp.jp (K.O.); sugihara.hiroki@omhp.jp (H.S.); \\ murakami.fumiyoahi@omhp.jp (F.M.); okamoto.shinya@omhp.jp (S.O.); hoshino.yoshinori@omhp.jp (Y.H.); \\ gotou.yuka.2@omhp.jp (Y.G.); takei.eisuke@omhp.jp (E.T.) \\ 2 Department of Surgery Onomichi Municipal Hospital, Shintakayama, Onomichi, Hiroshima 3-1170-177, \\ Japan; kimura.keisuke@omhp.jp (K.K.); onoda.tadashi@omhp.jp (T.O.) \\ 3 Faculty of Pharmacy and Pharmaceutical Sciences, Fukuyama University, Hiroshima 729-0292, Japan; \\ kban@fukuyama-u.ac.jp (K.B.); s.takeda@fukuyama-u.ac.jp (S.T.) \\ * Correspondence: sugihara@fukuyama-u.ac.jp; Tel.: +1-84-93-2111
}

Received: 7 October 2020; Accepted: 10 November 2020; Published: 11 November 2020

\begin{abstract}
Prognostic prediction has been reported to affect the decision of doctors and non-physician health care providers such as nurses, social workers, pastors, and hospice volunteers on the selection of appropriate medical interventions. This was a case of a 65-year-old woman who presented with a poor oral intake. The patient had a history of sigmoid colon cancer with abdominal wall metastasis and peritoneal dissemination. On the day of admission, nausea, anorexia, and malaise were noted, requiring immediate intervention. The patient's prognosis was predicted using the Palliative Prognostic Index. The pharmacist suggested the use of dexamethasone tablets in order to alleviate the patient's symptoms. Indeed, the administration of dexamethasone alleviated the symptoms of nausea, loss of appetite, and malaise. To the best of our knowledge, this is the first case report to demonstrate that prognosis prediction is important not only for other medical staff but also for pharmacists when deciding the need to initiate a treatment and continue such treatment, and when providing pharmacist interventions.
\end{abstract}

Keywords: pharmacist intervention; prognosis; Palliative Prognostic Index; terminal cancer patient

\section{Introduction}

Prognostic prediction has been reported to affect the decision of doctors and non-physician health care providers such as nurses, social workers, pastors, and hospice volunteers on appropriate medical interventions [1]. Morita et al. [2] reported that the long-term use of midazolam can increase the risk of developing drug tolerance; therefore, midazolam should only be used for patients with limited prognoses. Similarly, Morita et al. [3] reported that one liter or more of hydration per day could worsen peripheral edema, ascites, and pleural effusions in terminally ill cancer patients.

Traditionally, prognostic prediction has been based on the experience of clinicians. However, the development of objective prognostic prediction methods such as the Palliative Prognostic Index (PPI) [4] that can be used for terminally ill patients has made it possible for non-clinician medical staff to predict the patient's prognosis. Baba et al. [5] reported that the PPI is simple and highly feasible. PPI without blood tests is also feasible for pharmacists. However, to the best of our knowledge, no study has reported that prognostic prediction can affect the pharmacists' decision regarding the selection of appropriate medical interventions. 
In this paper, we describe a case in which a pharmacist predicted the prognosis of a terminal cancer patient using the PPI and performed pharmacist interventions. Here, we report that prognosis prediction is important for pharmacists in the selection of treatment.

\section{Case Presentation Section}

\subsection{Case Report}

A 65-year-old woman presented with a poor oral intake. She had no significant past medical history. She had sigmoid colon cancer with abdominal wall metastasis and peritoneal dissemination. She had been treated with loxoprofen sodium hydrate and hydromorphone hydrochloride. She used hydromorphone hydrochloride, as needed, as treatment for pain, and domperidone, as needed, as treatment for nausea and vomiting.

\subsection{Prognostic Predictive by PPI and Steroid Therapy}

The five variables used to determine the PPI are as follows: Palliative Performance Scale (PPS), oral intake, dyspnea at rest, delirium, and edema [4]. The PPS [6], a modification of the Karnofsky Performance Scale, measures ambulation, activity, selfcare, intake, and level of consciousness. A PPI score of 4 or less predicts a survival of more than 6 weeks, while a PPI score of 6 or more predicts a survival less than 3 weeks [4]. Administration of corticosteroids has been reported to be effective for symptoms such as nausea, anorexia, and malaise in advanced cancer patients [7-9]. In Japan, Matsuo et al. [10] reported that steroid therapy is often initiated within the prognosis of 1 to 2 months.

\subsection{Pharmacist Intervention}

On the day of admission (Day 1), grade 3 nausea, anorexia, and malaise were observed using the Common Terminology Criteria for Adverse Events (CTCAE) version 5.0. Based on the severity of symptoms, the patient required immediate management to alleviate these symptoms.

Therefore, the prognosis of this patient was predicted using the PPI. The patient's PPI was 3.5 (Table 1), and the prognosis was judged to be 1 to 2 months. Glare et al. [11] reported that the doctor's prognosis was longer than the actual prognosis. In this case, as in the case of Glare et al., the doctor may have overestimated the prognosis. For the above reasons, the pharmacist suggested the use of oral dexamethasone in order to alleviate the patient's symptoms. On Day 2, treatment with dexamethasone $2 \mathrm{mg} /$ day was started.

The day after the administration of dexamethasone (Day 3), the severity of nausea decreased from Grade 3 to Grade $0-1$, and the severity of anorexia and malaise decreased from Grade 3 to Grade 1 (Table 2). Based on the above results, the administration of dexamethasone was considered to alleviate the symptoms of nausea, loss of appetite, and malaise. She had a temporary mechanical ileus, but administration of dexamethasone $2 \mathrm{mg} /$ day was effective for about 2 weeks for nausea, anorexia, and malaise. However, since the effect gradually disappeared, treatment with dexamethasone $4 \mathrm{mg} /$ day was started on Day 23, but no effect was observed, and she died on Day 34. 
Table 1. Palliative Prognostic Index of our case.

\begin{tabular}{ccc}
\hline Variables & Partial Score & Partial Score of Our Case \\
\hline Palliative Performance Scale & 4 & 2.5 \\
$10-20$ & 2.5 & \\
$30-50$ & 0 & 1 \\
$\geq 60$ & 2.5 & \\
Oral intake & 1 & \\
Mouthful or less & 0 & \\
Normal & & \\
Reduced but more than mouthful & 3.5 & 0 \\
Dyspnea at rest & 0 & \\
Present & & \\
Absent & 4 & \\
Delirium & 0 & \\
Present & & \\
Absent & 1 & \\
Edema & 0 & \\
Present & & \\
Absent & &
\end{tabular}

Table 2. Subjective symptoms.

\begin{tabular}{lcc}
\hline & Before (Day 1) & After (Day 3) \\
\hline Nausea & Grade 3 & Grade 0-1 \\
\hline Anorexia & Grade 3 & Grade 1 \\
\hline Malaise & Grade 3 & Grade 1 \\
\hline
\end{tabular}

The severity of symptoms was evaluated using the Common Terminology Criteria for Adverse Events version 5.0.

\subsection{Ethical Consideration}

This study was approved by the research ethics committee of Onomichi Municipal Hospital (approval number 20-10, 23 September 2020).

\section{Discussion}

Here, we report a case in which the patient's nausea, anorexia, and malaise were alleviated by performing the pharmacist's interventions based on the results of the prediction of the terminal cancer patient's prognosis using the PPI. It is considered that the patient's quality of life (QOL) improved by alleviating the symptoms of nausea, loss of appetite, and malaise. To the best of our knowledge, this is the first case report to show that prognosis prediction is important not only for other medical staff, but also for pharmacists when deciding on the necessity of initiating a treatment and continuing such treatment, and when performing pharmacist interventions.

Mendis et al. [12] reported that prognosis prediction of multidisciplinary team can assist treating teams to recognize and articulate prognosis, facilitate treatment decisions, and plan end-of-life care appropriately. It is expected that prognosis prediction of pharmacists contributes to prognosis prediction of multidisciplinary team.

In the future, it is expected that it will become more common for pharmacists to predict the prognosis and perform pharmacist interventions. Besides, various tools for predicting the prognosis of terminal cancer patients have been developed in addition to the PPI. Moreover, new prognostic prediction tools that can be used for performing the appropriate pharmacist interventions will be developed. 


\section{Conclusions}

To the best of our knowledge, this is the first case report to show that prognosis prediction is important not only for other medical staff but also for pharmacists when deciding on the necessity of initiating treatments and continuing such treatments and performing pharmacist interventions.

Author Contributions: Conceptualization, methodology, formal analysis, investigation, and writing-original draft preparation, M.O. and K.O.; investigation and writing—review and editing, K.K., H.S., F.M., S.O., Y.H., Y.G., K.B., T.O., E.T., S.T., and N.S. All authors have read and agreed to the published version of the manuscript.

Funding: This research received no external funding.

Acknowledgments: We would like to thank Tomohiro Hosokawa for their assistance.

Conflicts of Interest: The authors declare no conflict of interest.

\section{References}

1. Steinhauser, K.E.; Christakis, N.A.; Clipp, E.C.; McNeilly, M.; Grambow, S.; Parker, J.; Tulsky, J.A. Preparing for the end of life: Preferences of patients, families, physicians, and other care providers. J. Pain Symptom Manag. 2001, 22, 727-737. [CrossRef]

2. Morita, T.; Tei, Y.; Inoue, S. Correlation of the dose of midazolam for symptom control with administration periods: The possibility of tolerance. J. Pain Symptom Manag. 2003, 25, 369-375. [CrossRef]

3. Morita, T.; Hyodo, I.; Yoshimi, T.; Ikenaga, M.; Tamura, Y.; Yoshizawa, A.; Shimada, A.; Akechi, T.; Miyashita, M.; Adachi, L.; et al. Association between hydration volume and symptoms in terminally ill cancer patients with abdominal malignancies. Ann. Oncol. 2005, 16, 640-647. [CrossRef] [PubMed]

4. Morita, T.; Tsunoda, J.; Inoue, S.; Chihara, S. The Palliative Prognostic Index: A scoring system for survival prediction of terminally ill cancer patients. Support. Care Cancer 1999, 7, 128-133. [CrossRef] [PubMed]

5. Baba, M.; Maeda, I.; Morita, T.; Inoue, S.; Ikenaga, M.; Matsumoto, Y.; Sekine, R.; Yamaguchi, T.; Hirohashi, T.; Tajima, T.; et al. Survival prediction for advanced cancer patients in the real world: A comparison of the Palliative Prognostic Score, Delirium-Palliative Prognostic Score, Palliative Prognostic Index and modified Prognosis in Palliative Care Study predictor model. Eur. J. Cancer 2015, 51, 1618-1629. [CrossRef] [PubMed]

6. Anderson, F.; Downing, G.M.; Hill, J.; Casorso, L.; Lerch, N. Palliative Performance Scale (PPS): A new tool. J. Palliat. Care 1996, 12, 5-11. [CrossRef] [PubMed]

7. Bruera, E.; Roca, E.; Cedaro, L.; Carraro, S.; Chacon, R. Action of oral methylprednisolone in terminal cancer patients: A prospective randomized double-blind study. Cancer Treat. Rep. 1985, 69, 751-754. [PubMed]

8. Mercadante, S.; Fulfaro, F.; Casuccio, A. The use of corticosteroids in home palliative care. Support. Care Cancer 2001, 9, 386-389. [CrossRef] [PubMed]

9. Yennurajalingam, S.; Frisbee-Hume, S.; Palmer, J.L.; Delgado-Guay, M.O.; Bull, J.; Phan, A.T.; Tannir, N.M.; Litton, J.K.; Reddy, A.; Hui, D.; et al. Reduction of cancer-related fatigue with dexamethasone: A double-blind, randomized, placebo-controlled trial in patients with advanced cancer. J. Clin. Oncol. 2013, 31, 3076-3082. [CrossRef] [PubMed]

10. Matsuo, N.; Morita, T.; Iwase, S. Physician-reported corticosteroid therapy practices in certified palliative care units in Japan: A nationwide survey. J. Palliat. Med. 2012, 15, 1011-1018. [CrossRef] [PubMed]

11. Glare, P.; Virik, K.; Jones, M.; Hudson, M.; Eychmuller, S.; Simes, J.; Christakis, N. A systematic review of physicians' survival predictions in terminally ill cancer patients. BMJ 2003, 327, 195-198. [CrossRef] [PubMed]

12. Mendis, R.; Soo, W.K.; Zannino, D.; Michael, N.; Spruyt, O. Multidisciplinary Prognostication Using the Palliative Prognostic Score in an Australian Cancer Center. Palliat. Care 2017, 9, 7-14. [CrossRef] [PubMed]

Publisher's Note: MDPI stays neutral with regard to jurisdictional claims in published maps and institutional affiliations.

(C) 2020 by the authors. Licensee MDPI, Basel, Switzerland. This article is an open access article distributed under the terms and conditions of the Creative Commons Attribution (CC BY) license (http://creativecommons.org/licenses/by/4.0/). 